\title{
DEGUM-Mitteilungen
}

\author{
Deutsche Gesellschaft für Ultraschall in der Medizin
}

\section{Bericht zur DEGUM-Pressekonferenz anlässlich des 40. Dreiländertreffens der DEGUM, ÖGUM und SGUM am 27. Oktober 2016 in Leipzig}

Unverzichtbares Multitalent: Ultraschall revolutioniert Diagnostik und Therapie - von Schwangerschaft bis Herz-OP

Die fortschreitende Technik hat in den vergangenen Jahren immer leistungsstärkere Ultraschallgeräte auf den Markt gebracht. Inzwischen erreichen die Bilder der HighTech-Präzisionsinstrumente sogar Mikroskop-Qualität. Trotzdem bevorzugen viele Ärzte weiterhin Untersuchungsmethoden wie die Computertomografie - wenngleich sich die Strahlenbelastung für Patienten kaum verringert hat. Ein Grund: „Obwohl die strahlenlose und nicht-invasive Ultraschall-Methode am schonendsten ist, bleibt den deutschen Ärzten die finanzielle Unterstützung dafür oft verwehrt", so Professor Dr. med Andreas Hagendorff, Sekretär der DEGUM auf einer Pressekonferenz in Leipzig anlässlich des 40. Dreiländertreffens der Deutschen Gesellschaft für Ultraschall in der Medizin e. V.(DEGUM) und ihrer österreichischen und schweizerischen Partnerorganisationen ÖGUM und SGUM.

„Im Vergleich zu niedergelassenen Ärzten in Österreich und der Schweiz stehen die deutschen Kollegen bei den Vergütungen für Ultraschalluntersuchungen am schlechtesten da“, so Hagendorff, der stellvertretender Leiter der Abteilung für Kardiologie und Angiologie am Universitätsklinikum Leipzig ist. „Wir haben mit dem bildgebenden Verfahren eine Methode, mit der wir exzellent arbeiten und ausbilden könnten.“ Mit dem Kongress wendeten sich die Fachgesellschaften insbesondere auch an den ärztlichen Nachwuchs in der Ultraschallmedizin. Neben einer eigens für Studenten ausgerichteten Einführung und Sitzungen zum Thema Ultraschall-Ausbildung bot die DEGUM auch in diesem Jahr wieder ein studentisches Anwenderseminar mit Theorie- und Praxisteilen an.

\section{Kostendruck motiviert Ärzte nicht zum ,Schallen“ $\nabla$}

Dr. med. Hans Worlicek von der Internistischen Praxis im Facharztzentrum Regensburg ergänzte, dass der steigende Kostendruck nicht besonders motiviert: „Der Arzt muss in moderne Geräte und seine Ausbildung investieren, aber erhält beispielsweise für die normale Bilduntersuchung des Bauchraumes nur etwa $14 €$.“

\section{Echokardiografie ermöglicht neue Wege in der Herzklappentherapie $\nabla$}

Auf der Pressekonferenz erläuterte Hagendorff, dass Ultraschall eine vorrangige Entscheidungshilfe dafür biete, ob man bei der Herzklappentherapie chirurgisch oder interventionell vorgeht. „Ultraschall

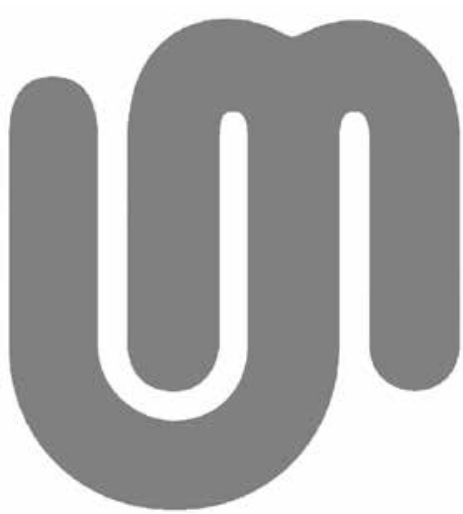

besitzt die beste räumliche Auflösung. Während man mit Ultraschall sozusagen Bilder malt, schießt man mit Computertomogramm (CT) oder Magnetresonanztomogramm (MRT) eher Fotos“, erläuterte der Experte. Bei Defekten an den Herzklappen, die per Katheter behoben werden können, empfiehlt Hagendorff deshalb während und nach dem Eingriff eine Kontrolle per Ultraschall.

\section{Ultraschall: bedeutendes Element in der Pränataldiagnostik $\nabla$}

Auch aus dem Fachgebiet der Pränataldiagnostik ist der Ultraschall heute nicht mehr wegzudenken, bestätigte DEGUMPräsident PD Dr. Kai-Sven Heling. Er betreibt in Berlin eine Praxis für Pränatalmedizin. „Laut einer aktuellen Analyse können durch die frühe Ultraschalluntersuchung des Fötus bereits rund die Hälfte der kindlichen Fehlbildungen erkannt werden." Aber auch diese als sogenanntes Ersttrimester-Screening bekannte Untersuchung wird von den deutschen Krankenkassen nicht übernommen und muss von den werdenden Eltern selbst bezahlt werden.

Die Ultraschalluntersuchung beim ungeborenen Kind wird beispielsweise eingesetzt, um Herzfehler zu erkennen. „Die Untersuchung ermöglicht uns eine frühzeitige Risikoabschätzung“, erklärte Pro-

\section{Geschäftsstelle}

Pressehaus/2209

Schiffbauerdamm 40

10117 Berlin

Tel.: +49 (0)30 $20608888-0$

Fax: +49 (0)30 $20608888-9$

E-Mail: geschaeftsstelle@degum.de Öffnungszeiten: Mo-Fr 8 bis 14 Uhr 
fessor Dr. med. Renaldo Faber vom Zentrum für Pränatale Medizin in Leipzig. Wenn ein Herzfehler diagnostiziert wird, müssten die Experten einschätzen, wie schwerwiegend dieser sei, ob das Kind kurz nach der Geburt operiert werden müsse, oder ob die Korrektur auf ein späteres Lebensalter verschoben werden könne. „Das Ergebnis dieser Ultraschalluntersuchung ist auch wichtig, um die
Geburt optimal zu planen und gegebenenfalls bestimmte Vorsichtsmaßnahmen für die Geburt vorzubereiten“, so der Experte weiter. 